\title{
Towards transdisciplinary education
}

\author{
BASARAB NICOLESCU ${ }^{1}$
}

\begin{abstract}
The methodology of transdisciplinarity is founded on three postulates: there are, in Nature and in our knowledge of Nature, different levels of Reality and, correspondingly, different levels of perception; the passage from one level of Reality to another is insured by the logic of the included middle; and the structure of the totality of levels of Reality or perception is a complex structure: every level is what it is because all the levels exist at the same time. After giving an exposition of these postulates the author contends that transdisciplinarity does not rest on a transfer from modern science. Instead, modern science, via its most general aspects, makes it possible to identify the postulates of transdisciplinarity. However, once they are formulated they have a much wider validity then in modern science itself, namely they could be applied in the field of education and culture.

It is argued that transdisciplinary education, founded on the transdisciplinary methodology, will allow scientists to establish links between persons, facts, images, representations, fields of knowledge and action.
\end{abstract}

Keywords. Transdisciplinarity, in vitro and in vivo knowledge, axiom of the included middle, transdisciplinary education.

\section{Multi, inter and transdisciplinarity}

The process of the decline of civilizations is one of enormous complexity and its roots lie deep in obscurity. Of course, one can find multiple after-the-fact explanations and rationalisations without ever successfully dissipating the feeling that there is an irrational element at work at the heart of the process. Neither the masses nor great decision makers, as actors in a well-defined civilisation, seem able to stop the decline of their civilization, even if they become more or less aware of the processes at work.

One thing is certain: this fall is always accompanied by a great unbalance between the mentalities of the actors and the inner developmental needs of a particular type of society. Although a civilization never stops proliferating new knowledge, it is as if these can never be fully

1 Theoretical physicist at CNRS, University Paris VI. Member of the Romanian Academy. President of the International Center for Transdisciplinary Research and Studies (CIRET). 
integrated within those who belong to this civilization. And it is the human being who must be placed at the centre of any civilization worthy of the name.

The unprecedented increase of knowledge in our era raises the challenging question of how to adapt our mentality to being. Mondialisation is today a potential source of a new decline. The two extreme dangers of mondialisation are, on one side, the cultural and spiritual homogenisation and, on another side, the paroxysm of ethnical and religious conflicts, as a self-defence reaction of different cultures and civilisations.

Harmony between inner being and outer knowledge presupposes that these known facts would be intelligible, comprehensible. But can such comprehension exist in the era of the disciplinary big bang and relentless specialization?

The indispensable need for bridges between the different disciplines is attested to by the emergence of multidisciplinarity and interdisciplinarity around the middle of the twentieth century.

Multidisciplinarity concerns studying a research topic not in just one discipline but in several at the same time. For example, a painting by Giotto can be studied not only within the context of art history, but also within the contexts of the history of religions, European history, or geometry. The topic in question will ultimately be enriched by incorporating the perspectives of several disciplines. Moreover, our understanding of the topic in terms of its own discipline is deepened by a fertile multidisciplinary approach. Multidisciplinarity brings a plus to the discipline in question (the history of art in our example), but we must remember that this "plus" is always in the exclusive service of the home discipline. In other words, the multidisciplinary approach overflows disciplinary boundaries while its goal remains limited to the framework of disciplinary research.

Interdisciplinarity has a different goal than multidisciplinarity. It concerns the transfer of methods from one discipline to another. One can distinguish three degrees of interdisciplinarity: (a) degree of application (for example, when the methods of nuclear physics are transferred to medicine, which leads to the appearance of new treatments for cancer); (b) epistemological degree (such as, transferring methods of formal logic to the area of general law, which generates some interesting analyses of the epistemology of law); (c) degree of the generation of new disciplines (when methods from mathematics are transferred to physics, generating mathematical physics, or when mathematical methods are transferred to meteorological phenomena or stock market processes, generating chaos theory; transferring methods from particle physics to astrophysics produces quantum cosmology. Like multidisciplinarity, interdisciplinarity overflows the disciplines, but its goal still remains within the framework of disciplinary research. 
As the prefix "trans" indicates, transdisciplinarity concerns that which is at once between the disciplines, across the different disciplines, and beyond all disciplines. ${ }^{2}$ Its goal is the understanding of the present world, of which one of the imperatives is the unity of knowledge. The word itself is quite recent: it was first introduced by Jean Piaget in $1970 .^{3}$

Is there something between and across the disciplines and beyond all disciplines? From the point of view of classical thought there is absolutely nothing. The space in question is empty, completely void, like the vacuum of classical physics.

In the presence of several levels of Reality, the space between disciplines and beyond disciplines is full, just as the quantum void is full of all potentialities: from the quantum particle to the galaxies, from the quark to the heavy elements that condition the appearance of life in the universe.

Transdisciplinary research is clearly distinct from disciplinary research, even while being entirely complementary. Disciplinary research concerns, at most, one and the same level of Reality; moreover, in most cases, it only concerns fragments of one level of Reality. In contrast, transdisciplinarity concerns the dynamics engendered by the action of several levels of Reality at once. The discovery of these dynamics necessarily passes through disciplinary knowledge.

Disciplinarity, multidisciplinarity, interdisciplinarity, and transdisciplinarity are like four arrows shot from but a single bow: knowledge.

The transdisciplinary knowledge TK, corresponds to a new type of knowledge - in vivo knowledge. This new knowledge is concerned with the correspondence between the external world of the Object and the internal world of the Subject. By definition, the TK knowledge includes a system of values (see Table I).

It is important to realize that the disciplinary knowledge and the transdisciplinary knowledge are not antagonist but complementary. Both their methodologies are founded on scientific attitude. The new, transdisciplinary knowledge engenders a new, transdisciplinary education. In order to explore what this means, we have to understand what could be the transdisciplinary methodology.

2 B Nicolescu, La transdisciplinarité, manifeste, (Le Rocher, Monaco, Collection "Transdisciplinarité", 1996); English translation: Manifesto of Transdisciplinarity, (Translated from the French by Karen-Claire Voss, SUNY Press, New York, 2002).

$3 \mathrm{~J}$ Piaget, L'épistémologie des relations interdisciplinaires, in L'interdisciplinarité - Problèmes d'enseignement et de recherche dans les universités, (OCDE, Paris, 1972, proceedings of a workshop held in Nice in 1970). 


\begin{tabular}{|l|l|}
\hline KNOWLEDGE DK & $\begin{array}{l}\text { KNOWLEDGE TK } \\
\text { IN VITRO }\end{array}$ \\
\hline External world - Object & $\begin{array}{l}\text { Correspondence between external } \\
\text { world (Object) and } \\
\text { internal world (Subject) }\end{array}$ \\
\hline knowledge & understanding \\
\hline analytic intelligence & $\begin{array}{l}\text { new type of intelligence - harmony be- } \\
\text { tween mind, feelings and body }\end{array}$ \\
\hline $\begin{array}{l}\text { oriented towards power and posses- } \\
\text { sion }\end{array}$ & $\begin{array}{l}\text { oriented towards astonishment and } \\
\text { sharing }\end{array}$ \\
\hline binary logic & included middle logic \\
\hline exclusion of values & inclusion of values \\
\hline
\end{tabular}

Table 1. Comparison between disciplinary knowledge DK and transdisciplinary knowledge TK.

\section{The transdisciplinary approach of Nature and knowledge}

The transdisciplinary approach of Nature and knowledge can be described through the diagram shown in Fig. 1.

In the left part are symbolically drawn the levels of Reality

$\left\{\mathrm{NR}_{\mathrm{n}}, \ldots, \mathrm{NR}_{2}, \mathrm{NR}_{1}, \mathrm{NR}_{0}, \mathrm{NR}_{-1}, \mathrm{NR}_{-2}, \ldots, \mathrm{NR}_{-\mathrm{n}}\right\}$

The index $\mathrm{n}$ can be finite or infinite.

Here the meaning we give to the word "reality" is pragmatic and ontological at the same time.

By "Reality" (with a capital "R") we intend first of all to designate that which resists our experiences, representations, descriptions, images, or even mathematical formulations.

Insofar as Nature participates in the being of the world, one must give an ontological dimension to the concept of Reality. Reality is not merely a social construction, the consensus of a collectivity, or some intersubjective agreement. It also has a trans-subjective dimension: e.g. experimental data can ruin the most beautiful scientific theory.

Of course, one has to distinguish the word "Real" and "Reality". Real designates that what it is, while Reality is connected to resistance in our human experience. The "Real" is, by definition, veiled for ever, while the "Reality" is accessible to our knowledge.

By "level of Reality", notion I first introduced in Nous, la particule et le 
monde ${ }^{4}$ and later developed in other works, ${ }^{5}$ I designate an ensemble of systems which are invariant under certain laws: for example, quantum entities are subordinate to quantum laws, which depart radically from the laws of the physical world. That is to say that two levels of Reality are different if, while passing from one to the other, there is a break in the laws and a break in fundamental concepts (like, for example, causality).

Levels of Reality are radically different from levels of organization as these have been defined in systemic approaches. Levels of organization do not presuppose a break with fundamental concepts: several levels of organization can appear at one and the same level of Reality. The levels of organization correspond to different structures of the same fundamental laws. For example, Marxist economy and classical physics belong to one and the same level of Reality.

The emergence of at least three different levels of Reality in the study of natural systems - the macrophysical level, the microphysical level and the cyber-space-time - is a major event in the history of knowledge. It can lead us to reconsider our individual and social lives, to give a new interpretation to old knowledge, to explore the knowledge of ourselves in a different way, here and now.

The existence of different levels of Reality has been affirmed by different traditions and civilizations, but this affirmation was founded either on religious dogma or on the exploration of the interior universe only.

In our century, in an effort to question the foundations of science, Edmund Husser ${ }^{6}$ and other scholars have detected the existence of different levels of perception by the subject-observer of Reality.

The transdisciplinary viewpoint allows us to consider a multidimensional Reality, structured by multiple levels replacing the single-level, one-dimensional reality of classical thought.

According to the transdisciplinary approach, Reality is structured via a certain number of levels. The considerations which follow do not depend

4 B Nicolescu, Nous, la particule et le monde, (2e édition, Le Rocher, Monaco, Collection "Transdisciplinarité", 2002).

5 B Nicolescu, "Levels of complexity and levels of reality", in B Pullman (ed.), The emergence of complexity in mathematics, physics, chemistry, and biology. Proceedings of the plenary session of the Pontifical Academy of Sciences, 27-31 October 1992, (Casina Pio IV, Vatican, Ed. Pontificia Academia Scientiarum, Vatican City, 1996, Distributed by Princeton University Press); B Nicolescu, "Gödelian aspects of nature and knowledge" in G Altmann and WA Koch (eds.), Systems - new paradigms for the human sciences, (Walter de Gruyter, Berlin - New York, 1998); M Camus, T Magnin, B Nicolescu and K-C Voss, "Levels of representation and levels of reality: towards an ontology of science" in NH Gregersen, MWS Parsons and C Wassermann (eds.), The concept of nature in science and theology, II, (Éditions Labor et Fides, Genève, 1998), pp. 94-103; B Nicolescu, Hylemorphism, quantum physics and levels of reality, in Demetra Sfendoni-Mentzou, introduction by Hilary Putnam (eds.), Aristotle and contemporary science, I, (Peter Lang, New York, 2000), pp. 173-184.

6 E Husserl, Méditations cartésiennes, (Translated form German by Gabrielle Peiffer and Emmanuel Levinas, Vrin, Paris, 1966). 
on whether or not this number is finite or infinite. For the sake of clarity, let us suppose that this number is infinite (i.e. we take $n \rightarrow \infty$ in Fig. 1).

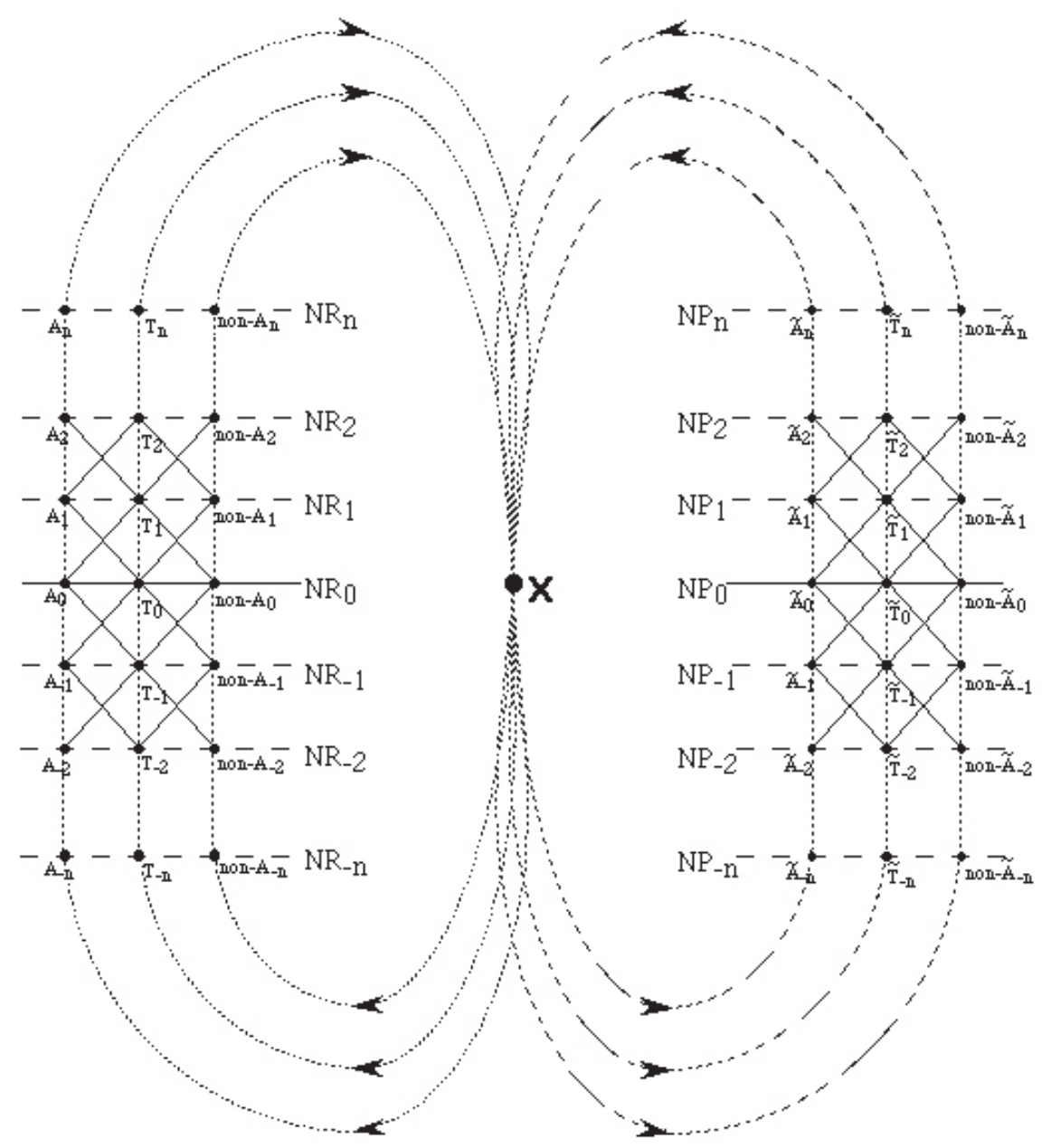

Figure 1: The transdisciplinary Object, the transdisciplinary subject and Interaction term.

Two adjacent levels in Fig. 1 (say, $\mathrm{NR}_{0}$ and $\mathrm{NR}_{1}$ ) are connected by the logic of the included middle, a new logic as compared with classical logic.

The classical logic is founded on three axioms:

1. The axiom of identity: A is A.

2. The axiom of non-contradiction: A is not non-A.

3. The axiom of the excluded middle: There exists no third term $\mathrm{T}$ ("T" from "third") which is at the same time A and non-A. 
Using this logic one immediately arrives at the conclusion that the pairs of contradictories advanced by quantum physics are mutually exclusive, because one cannot affirm the validity of an assertion and of its opposite at the same time: A and non-A.

Most quantum logics ${ }^{7}$ have modified the second axiom of classical logic the axiom of non-contradiction - by introducing non-contradiction with several truth values in place of the binary pair (A and non-A). History will credit Stéphane Lupasco (1900-1988) with having shown that the logic of the included middle is a true logic, formalisable and formalized, multivalent (with three values: A, non-A, and T) and non-contradictory. ${ }^{8}$

Our understanding of the axiom of the included middle - there exists a third term $\mathrm{T}$ which is at the same time A and non-A - is completely clarified once the notion of "levels of Reality" is introduced.

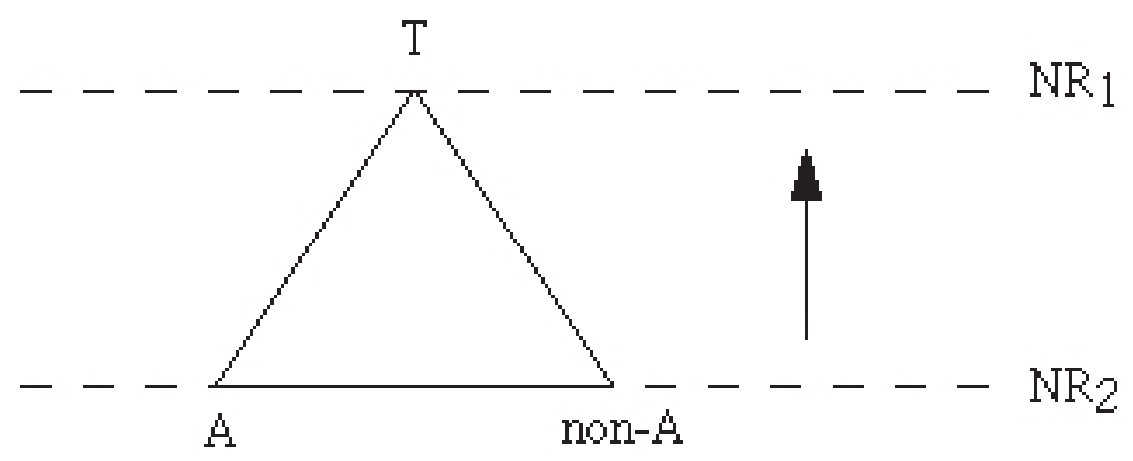

In order to obtain a clear image of the meaning of the included middle, we represent in Fig. 2 the three terms of the new logic - A, non-A, and T - and the dynamics associated with them by a triangle in which one of the vertices is situated at one level of Reality and the two other vertices at another level of Reality. The included middle is in fact an included third. If one remains at a single level of Reality, all manifestation appears as a struggle between two contradictory elements. The third dynamic, that of the T-state, is exercised at another level of Reality, where that which appears to be disunited is in fact united, and that which appears contradictory is perceived as non-contradictory.

It is the projection of the T-state onto the same single level of Reality which produces the appearance of mutually exclusive, antagonistic pairs (A and non-A). A single level of Reality can only create antagonistic oppo-

7 TA Brody, "On quantum logic", in Foundation of Physics, 14,(5), 1984, pp. 409-430.

8 S Lupasco, Le principe d'antagonisme et la logique de l'énergie, (2nd edition, Le Rocher, Paris, 1987), Foreword by B Nicolescu; Stéphane Lupasco - L'homme et l'oeuvre, Le Rocher, Monaco, coll. "Transdisciplinarité", 1999, under the direction of Horia Badescu and Basarab Nicolescu. 
sitions. It is inherently self-destructive if it is completely separated from all the other levels of Reality. A third term which is situated at the same level of Reality as that of the opposites A and non-A, cannot accomplish their reconciliation.

The $\mathrm{T}_{1}$-state present at the level $\mathrm{NR}_{1}$ (see Fig. 1) is connected to a pair of contradictories $\left(A_{0}\right.$ and non- $\left.A_{0}\right)$ at an immediately adjacent level. The $T_{1}$-state allows the unification of contradictories $A_{0}$ and non- $A_{0}$, but this unification takes place at a level different from the one $\mathrm{NR}_{0}$ on which $\mathrm{A}_{0}$ and non- $\mathrm{A}_{0}$ are situated. The axiom of non-contradiction is thereby respected.

There is certainly a coherence among different levels of Reality, at least in the natural world. In fact, an immense self-consistency - a cosmic bootstrap - seems to govern the evolution of the universe, from the infinitely small to the infinitely large, from the infinitely brief to the infinitely long. A flow of information is transmitted in a coherent manner from one level of Reality to another in our physical universe.

The logic of the included middle is capable of describing the coherence among these levels of Reality by an iterative process defined by the following stages: (1) A pair of contradictories $\left(A_{0}\right.$, non- $\left.A_{0}\right)$ situated at a certain level $\mathrm{NR}_{0}$ of Reality is unified by a $\mathrm{T}_{1}$-state situated at a contiguous level $\mathrm{NR}_{1}$ of Reality; (2) In turn, this $\mathrm{T}_{1}$-state is linked to a couple of contradictories $\left(A_{1}\right.$, non- $\left.A_{1}\right)$, situated at its own level; (3) The pair of contradictories $\left(A_{1}\right.$, non- $\left.A_{1}\right)$ is, in its turn, unified by a $T_{2}$-state situated at a third level $\mathrm{NR}_{2}$ of Reality, immediately contiguous to the level $\mathrm{NR}_{1}$ where the ternary $\left(A_{1}\right.$, non- $\left.A_{1}, T_{1}\right)$ is found. The iterative process continues to indefinitely until all the levels of Reality, known or conceivable, are exhausted.

In other words, the action of the logic of the included middle on the different levels of Reality induces an open structure of the unity of levels of Reality. This structure has considerable consequences for the theory of knowledge because it implies the impossibility of a self-enclosed complete theory. Knowledge is forever open.

The open structure of the unity of levels of Reality is in accord with one of the most important scientific results of the twentieth century concerning arithmetic, the theorem of Kurt Gödel, ${ }^{9}$ which states that a sufficiently rich system of axioms inevitably leads to results which are either indecisive or contradictory. The implications of Gödel's theorem have considerable importance for all modern theories of knowledge, primarily because it concerns not just the field of arithmetic, but all of mathematics which include arithmetic.

To be sure, there is a coherence of the unity of levels of Reality, but this coherence is oriented. If coherence is limited only to the levels of Real-

9 See, for example, E Nagel and JR Newman, Gödel's proof, (New York University Press, New York, 1958); Hao Wang, A logical journey - From Gödel to philosophy, (The MIT Press, Cambridge, Massachusetts - London, England, 1996). 
ity, it stops both at the "highest" level and at the "lowest" level (see Fig. 1 ). If we wish to suggest the idea of a coherence which continues beyond these two limiting levels, so that there is an open unity, we must conceive the unity of levels of Reality as a unity that extends by a zone of non-resistance to our experiences, representations, descriptions, images, and mathematical formulations. This zone of non-resistance corresponds to the "veil" which Bernard d'Espagnant referred to as "the veil of the Real". ${ }^{10}$ In this zone there are no levels of Reality.

Quite simply, the non-resistance of this zone of absolute transparence is due to the limitations of our bodies and of our sense organs - limitations which apply regardless of what measuring tools are used to extend these sense organs. The zone of non-resistance corresponds to the sacred - to that which does not submit to any rationalization. It is rational but not rationalizable, a distinction often used by Edgar Morin. ${ }^{11}$

It is important to note that the three loops of coherence in Fig. 1 are situated not only in the zone where the levels of Reality are absent but also in between the levels of Reality: the zone of non-resistance of the sacred penetrates and crosses the levels of Reality. In other words, the transdisciplinary approach of Nature and knowledge offers a link between the Real and the Reality.

The unity of levels of Reality and its complementary zone of nonresistance constitutes what we call the transdisciplinary Object.

A new Principle of Relativity ${ }^{12}$ [1] emerges from the coexistence between complex plurality and open unity: no level of Reality constitutes a privileged place from which one is able to understand all the other levels of Reality. A level of Reality is what it is because all the other levels exist at the same time. This Principle of Relativity is what originates a new perspective on religion, politics, art, education, and social life. And when our perspective on the world changes, the world changes. "Saying a true word is equivalent to the transformation of the world" - writes the great brazilian educator Paulo Freire in his Pedagogy of the Oppressed. ${ }^{13}$

The different levels of Reality are accessible to human knowledge thanks to the existence of different levels of perception, described diagrammatically at the right of Fig. 1. They are found in a one-to-one correspondence with levels of Reality. These levels of perception

$\left\{\mathrm{NP}_{\mathrm{n}}, \ldots, \mathrm{NP}_{2}, \mathrm{NP}_{1}, \mathrm{NP}_{0}, \mathrm{NP}_{-1}, \mathrm{NP}_{-2}, \ldots, \mathrm{NP}_{-\mathrm{n}}\right\}$

permit an increasingly general, unifying, encompassing vision of Reality,

10 B d'Espagnat, Le réel voilé - Analyse des concepts quantiques, (Fayard, Paris, 1994).

11 E Morin, La méthode III - La connaissance de la connaissance/1. Anthropologie de la connaissance, (Seuil, Paris, 1986).

12 B Nicolescu, La transdisciplinarité, manifeste, (Le Rocher, Monaco, Collection "Transdisciplinarité", 1996); English translation: Manifesto of Transdisciplinarity, (Translated from the French by Karen-Claire Voss, SUNY Press, New York, 2002).

13 P Freire, Pedagogy of the oppressed, (The Seabury Press, New York, 1968). 
without ever entirely exhausting it.

As in the case of levels of Reality, the coherence of levels of perception presuppose a zone of non-resistance to perception. In this zone there are no levels of perception.

The unity of levels of perception and this complementary zone of nonresistance constitutes what we call the transdisciplinary Subject.

The two zones of non-resistance of transdisciplinary Object and Subject must be identical for the transdisciplinary Subject to communicate with the transdisciplinary Object. A flow of consciousness that coherently cuts across different levels of perception must correspond to the flow of information coherently cutting across different levels of Reality. The two flows are interrelated because they share the same zone of non-resistance.

Knowledge is neither exterior nor interior: it is simultaneously exterior and interior. The studies of the universe and of the human being sustain one another.

The open unity between the transdisciplinary Object and the transdisciplinary Subject is conveyed by the coherent orientation of the flow of information, described by the three oriented loops in Fig. 1which cut through the levels of Reality, and of the flow of consciousness, described by the three oriented loops which cut through the levels of perception.

The loops of information and consciousness have to meet in a least one point $\mathrm{X}$ in order to insure the coherent transmission of information and consciousness everywhere in the visible and invisible regions of the Universe. In some sense, the point $X$ is the source of Reality and perception. The point $\mathrm{X}$ and its associated loops of information and consciousness describe the third term of the transdisciplinary knowledge: the Interaction term between the Subject and the Object, which can not be reduced neither to the Object nor to the Subject.

This ternary partition

$$
\text { \{ Subject, Object, Interaction \} }
$$

is radically different from the binary partition

\{ Subject, Object \}

which defines the modern metaphysics.

The view I am expressing here is totally conform to the one of the founders of quantum mechanics Werner Heisenberg, Wolfgang Pauli and Niels Bohr.

In fact, Werner Heisenberg came very near, in his philosophical writings, to the concept of "level of Reality". In his famous Manuscript of the year 1942 (published only in 1984) Heisenberg, who knew well Husserl, introduces the idea of three regions of reality, able to give access to the 
concept of "reality" itself: the first region is that of classical physics, the second - of quantum physics, biology and psychic phenomena and the third - that of the religious, philosophical and artistic experiences. ${ }^{14}$ This classification has a subtle ground: the closer and closer connectiveness between the Subject and the Object.

The methodology of transdisciplinarity is therefore founded on three postulates:

i. There are, in Nature and in our knowledge of Nature, different levels of Reality and, correspondingly, different levels of perception.

ii. The passage from one level of Reality to another is insured by the logic of the included middle.

iii. The structure of the totality of levels of Reality or perception is a complex structure: every level is what it is because all the levels exist at the same time. ${ }^{15}$

The first two get their experimental evidence from quantum physics, while the last one has its source not only in quantum physics but also in a variety of other exact and human sciences.

It is important to note that one can assume the validity of the three postulates of transdisciplinarity independently of their historical roots in some branches of modern science. In other words transdisciplinarity does not rest on a transfer from modern science: this would be a wrong epistemological and philosophical procedure. Modern science, via its most general aspects, allowed us to identify the three postulates of transdisciplinarity, but once they are formulated they have a much wider validity then in modern science itself, namely they could be applied in the field of education and culture.

The transdisciplinary education, founded on the transdisciplinary methodology, will allow us to establish links between persons, facts, images, representations, fields of knowledge and action, to discover the Eros of learning during our entire life and to built beings in permanent questioning and permanent integration.

14 W Heisenberg, Philosophie - Le manuscrit de 1942, (Translation from German and introduction by Catherine Chevalley, Seuil, Paris, 1998).

15 B Nicolescu, La transdisciplinarité, manifeste, (Le Rocher, Monaco, Collection "Transdisciplinarité", 1996); English translation: Manifesto of Transdisciplinarity, (Translation from the French by Karen-Claire Voss, SUNY Press, New York, 2002). 